\title{
Resistant hypercalcaemia in metastatic parathyroid carcinoma
}

\author{
We report a case of inoperable metastatic parathyroid carcinoma involving life- \\ threatening hypercalcaemia that failed to respond to standard therapy. Our review of \\ available therapeutic modalities showed a paucity of evidence to guide management \\ of patients with these rare tumours. This is the first report of the treatment of \\ malignant hypercalcaemia secondary to parathyroid carcinoma using denosumab.
}

\section{Samantha E Bowyer MB BCh, MRCP Medical Oncology Advanced Trainee \\ Alison M White MB BS, MPH, Medical Oncology Trainee 2 \\ David T Ransom MB BS, FRACP. Medical Oncologist \\ John A Davidson MB BS, FRACP, MBiostats Head of Department \\ 1 Department of Medical Oncology, Royal Perth Hospital, Perth, WA \\ 2 Department of Medical Oncology, Sir Charles Gairdner Hospital, Perth, WA \\ samantha.bowyer@ health.wa.gov.au}

doi: 10.5694/mjal2.11243

\section{Clinical record}

A 45-year-old man with no significant past medical history presented with a fractured clavicle and evidence of a radiolucent lesion (brown tumour) within the clavicle on $\mathrm{x}$ ray, characteristic for hyperparathyroidism (Box 1). On further investigation, biochemical analysis showing raised serum calcium and parathyroid hormone (PTH) levels confirmed primary hyperparathyroidism. A thyroid ultrasound showed a $39 \times 29 \times 32 \mathrm{~mm}$ mass in the right lobe, suggestive of a tumour. A staging computed tomography (CT) scan of the neck and chest and a bone scan showed bone changes consistent with hyperparathyroidism throughout the axial and appendicular skeleton. There was no evidence of metastatic disease.

The patient underwent a partial parathyroidectomy and right hemithyroidectomy. Histopathological examination confirmed parathyroid carcinoma. The tumour was invading the thyroid gland and there was evidence of lympho-

\section{Plain $x$-ray showing brown tumour at the distal end of the left clavicle, characteristic of hyperparathyroidism}

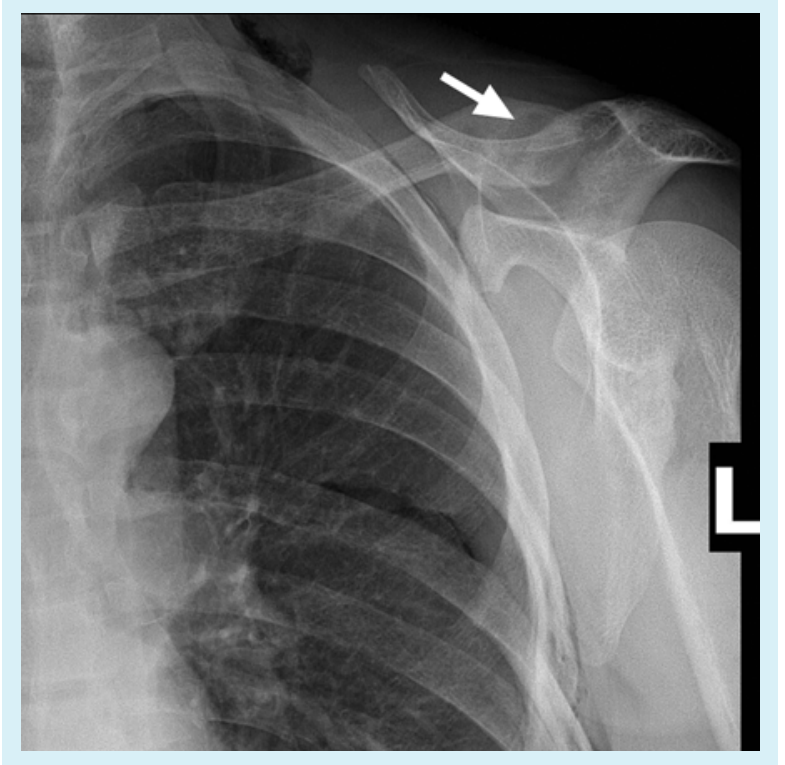

vascular invasion. After the operation, the patient developed persistent hypocalcaemia as a consequence of relative hypoparathyroidism. He had an ionised calcium level of $0.68 \mathrm{mmol} / \mathrm{L}$ (reference interval [RI], 1.12$1.32 \mathrm{mmol} / \mathrm{L}$ ). He was diagnosed with hungry bone syndrome, a common problem following parathyroidectomy. This required treatment with high-dose oral calcium (5.4 g daily) and calcitriol ( $0.25 \mu \mathrm{g}$ three times daily). Residual tumour was suspected, as his postoperative PTH level remained elevated, at $13.5 \mathrm{pmol} / \mathrm{L}$ (RI, $0.7-7.0 \mathrm{pmol} / \mathrm{L}$ ). A whole-body sestamibi scan (Tc [technetium]-99m MIBI [methoxyisobutylisonitrile]-830MBq) did not confirm this. He remained under surveillance with 4-monthly clinical review with blood tests and annual sestamibi scans.

One year after his surgery, the patient's PTH level had risen further, to $52.7 \mathrm{pmol} / \mathrm{L}$. His calcium supplements were ceased, as he was hypercalcaemic (serum corrected calcium, $3.11 \mathrm{mmol} / \mathrm{L}$; RI, 2.15-2.60 mmol/L). Despite ceasing supplementation, his hypercalcaemia worsened over 2 months, to a level of $4.15 \mathrm{mmol} / \mathrm{L}$, with a PTH level of $203 \mathrm{pmol} / \mathrm{L}$ and creatinine level of $166 \mu \mathrm{mol} / \mathrm{L}$ (RI, 60 $110 \mu \mathrm{mol} / \mathrm{L})$. On clinical examination, he had two small palpable nodules in the right thyroid bed. A CT scan of the neck confirmed a $1.5 \times 2.4 \times 1.6 \mathrm{~cm}$ mass at the site of the previous surgery.

A positron emission tomography scan showed mildly increased fludeoxyglucose activity at the right thyroid bed, consistent with low-volume residual disease, and intense activity in the left retrocrural soft tissue (Box 2). Biopsy specimens were obtained from both sites and were found to be histologically consistent with parathyroid cancer. The patient underwent a planned resection of the known sites of disease, beginning with a right-sided neck dissection. Thereafter, he underwent a left thoracotomy, which showed more extensive pleural deposits and bulky metastatic disease over the diaphragm and crura, only amenable to debulking. A left pleural effusion, non-malignant on cytology, complicated his postoperative recovery.

After surgery, his PTH level remained elevated, at $112 \mathrm{pmol} / \mathrm{L}$. His hypercalcaemia was difficult to manage despite using intravenous hydration and intravenous zoledronic acid (4 mg). His serum calcium level transiently fell from $3.14 \mathrm{mmol} / \mathrm{L}$ to $2.77 \mathrm{mmol} / \mathrm{L}$. Cinacalcet $30 \mathrm{mg}$ 
2 Positron emission tomography scan showing fludeoxyglucose avidity at the left retrocrural soft tissue, consistent with metastatic disease

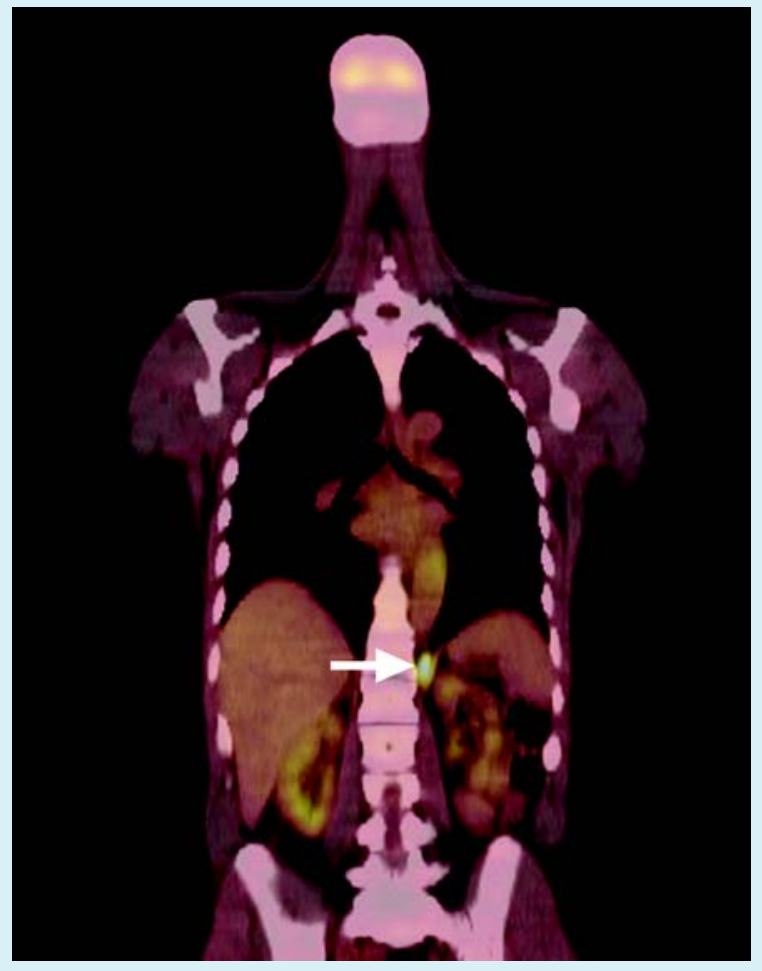

twice daily was commenced and titrated to a maximal dose of $90 \mathrm{mg}$ four times daily, with no significant improvement in serum calcium levels.

Given the patient's failure to respond to cinacalcet, in an attempt to reduce tumour size and function, he received two cycles of chemotherapy with a 3-weekly schedule of cyclophosphamide $500 \mathrm{mg} / \mathrm{m}^{2}$ on Day 1, 5-fluorouracil $500 \mathrm{mg} / \mathrm{m}^{2}$ daily on Days $1-4$ and dacarbazine $200 \mathrm{mg} / \mathrm{m}^{2}$ on Days 1-4. On presentation for Cycle 2, his calcium level was $4.37 \mathrm{mmol} / \mathrm{L}$ and PTH level $335 \mathrm{pmol} / \mathrm{L}$, indicating no biochemical response. He had symptomatic hypercalcaemia with fatigue, polydipsia, anorexia and renal dysfunction for management. He continued to receive weekly bisphosphonate infusions for management, but, despite this, his serum calcium levels did not fall below $3.0 \mathrm{mmol} / \mathrm{L}$.

We trialled denosumab as a means of achieving control of the resistant hypercalcaemia. We used loading doses, aiming to bring about rapid blockade of receptor activator of nuclear factor kappa B (RANK) ligand. The schedule was taken from a Phase II study of giant-cell tumours of bone, in which loading doses of denosumab $120 \mathrm{mg}$ were given subcutaneously on Days 1, 8 and 15, with monthly maintenance therapy thereafter. ${ }^{1}$ In our patient, after the initial loading dose, the serum-corrected calcium level dropped to $2.66 \mathrm{mmol} / \mathrm{L}$ by Day 8 . After a second dose, by Day 15 his calcium level was normal, at $2.33 \mathrm{mmol} / \mathrm{L}$. Calculated urinary N-telopeptide levels were monitored to test for suppression of bone turnover. The baseline level was $431 \mathrm{nmol}$ bone collagen equivalents (BCE)/mmol creatinine (RI, $<65 \mathrm{nmol} \mathrm{BCE} / \mathrm{mmol}$ creatinine); by Day 15 it fell to $<20 \mathrm{nmol} \mathrm{BCE} / \mathrm{mmol}$ creatinine, indicating rapid suppression of bone turnover. After two loading doses of denosumab, the patient's serum calcium remained within the normal range for 4 months. His creatinine level also decreased to $102 \mu \mathrm{mol} / \mathrm{L}$. Further treatment with denosumab was to be guided by the serum calcium level.

Despite control of the calcium level, the patient's disease progressed and his PTH level rose to a maximum of $433 \mathrm{pmol} / \mathrm{L}$. He became symptomatic, with a recurrent pleural effusion, anorexia and weight loss. Owing to a paucity of data on effective chemotherapeutic agents, the case was discussed at a departmental case review meeting and a regimen of carboplatin and gemcitabine was suggested, given its broad activity and use in carcinoma of unknown primary site. ${ }^{2}$ The patient completed six cycles of treatment with relative stability of the pleural effusion and retrocrural disease; however, his PTH level did not decrease. His physical condition deteriorated over 6 months after completion of chemotherapy, and he required one further dose of denosumab for calcium control. The patient died 1 year after his presentation with metastatic disease.

\section{Discussion}

Parathyroid carcinoma is rare, accounting for less than 1\% of cases of hyperparathyroidism. It is associated with a high rate of local and distant recurrence. The mainstay of treatment is surgical resection, even in metastatic disease. The disease is often refractory to medical management, leading to morbidity and mortality due to hypercalcaemia rather than metastatic disease; thus, an aggressive approach to resection has been advocated. ${ }^{3}$ Managing lifethreatening hypercalcaemia and controlling a relatively indolent tumour pose a significant therapeutic challenge in inoperable disease. ${ }^{4}$

The efficacy of cinacalcet in the management of parathyroid cancer-related hypercalcaemia was assessed in an open-label single-arm study of 29 patients. ${ }^{5}$ Cinacalcet was commenced at $30 \mathrm{mg}$ twice daily and titrated to $90 \mathrm{mg}$ four times daily or until there was a response in the serum calcium. Over the 16 -week study period, $62 \%$ of patients responded to therapy; the greatest response was seen in patients with the highest baseline calcium levels. PTH levels were also shown to decrease, but this was not clinically significant.

There is limited evidence for chemotherapy in the management of metastatic parathyroid carcinoma. Success using cyclophosphamide, 5-fluorouracil and dacarbazine has been reported, with normalisation of serum calcium and a complete response in pulmonary metastases after 12 cycles. ${ }^{6}$ There are several other case reports of agents that have demonstrated some efficacy, including dacarbazine as a single agent, the "MACC" regimen (methotrexate, adriamycin [doxorubicin], cyclophosphamide and CCNU [cyclonexyl-chloroethyl-nitrosourea, or lomustine]) and synthetic oestrogen therapy. ${ }^{4}$

Few strategies to manage resistant hypercalcaemia in parathyroid carcinoma have been published. One case report described immunisation using the bioactive section of PTH in a patient with resistant hypercalcaemia, with rapid improvement in symptoms and serum calcium. 


\section{Case reports}

Autoantibodies to parathyroid hormone were identified in the patient's serum within 4 weeks. The authors theorised that they prevented the binding of PTH to its receptors, thereby improving hypercalcaemia. There was no clinical or radiological evidence of tumour regression after 6 months of therapy. ${ }^{7}$

Denosumab is a fully human monoclonal IgG2 antibody that has a high affinity and specificity to bind RANK ligand, mimicking the effect of osteoprotegerin, which acts to competitively inhibit the binding of RANK ligand and RANK to inhibit osteoclast activation. ${ }^{8}$ Denosumab has been compared with zoledronic acid in the prevention of skeletal-related events, such as fractures, in patients with advanced malignancies in two randomised trials. In both, denosumab was associated with higher rates of hypocalcaemia and greater suppression of bone turnover markers. ${ }^{9,10}$ It is currently licensed in Australia for the treatment of osteoporosis and prevention of skeletal-related events in patients with metastatic breast and prostate cancer with bone metastases.

Preclinical data have shown that telomerase is active in parathyroid cancer cells and could be a potential therapeutic target. An in-vitro study of zidovudine, an antiretroviral agent, investigated its use in inhibiting telomerase. Zidovudine accumulated in malignant parathyroid cells in culture, which induced apoptosis. The effect was not observed in the adenomatous parathyroid cells, suggesting this could be considered as a targeted therapy. ${ }^{11}$ Studies in humans have not yet been done.

Metastatic parathyroid cancer is a rare malignancy that has a limited evidence base to guide management. We report a patient with refractory hypercalcaemia that dramatically responded to the RANK ligand inhibitor denosumab, with rapid suppression of bone turnover within days. Unlike bisphosphonates, denosumab does not require dose adjustment for renal impairment, which is common in patients with hypercalcaemia. Denosumab offered prolonged calcium control with less frequent administration. The management of parathyroid cancer remains a therapeutic challenge, owing to the minimal evidence for effective systemic therapy in patients with inoperable disease. We propose that denosumab should be considered in the treatment algorithm for patients with hypercalcaemia secondary to parathyroid cancer.

Competing interests: No relevant disclosures.

Received 9 Aug 2012, accepted 11 Feb 2013.

1 Thomas D, Henshaw R, Skubitz K, et al. Denosumab in patients with giant-cell tumour of bone: an open-label, phase 2 study. Lancet Oncol 2010; 11: 275-280.

2 Pittman KB, Olver IN, Koczwara B, et al. Gemcitabine and carboplatin in carcinoma of unknown primary site: a phase 2 Adelaide Cancer Trials and Education Collaborative study. Br J Cancer 2006; 95: 1309-1313.

3 Mezhir JJ, Melis M, Headley RC, et al. Successful palliation of hypercalcemia secondary to metastatic parathyroid cancer: an unusual indication for hepatic resection. J Hepatobiliary Pancreat Surg 2007; 14: 410-413.

4 Shane E. Clinical review 122: Parathyroid carcinoma. J Clin Endocrinol Metab 2001; 86: 485-493.

5 Silverberg SJ, Rubin MR, Faiman C, et al. Cinacalcet hydrocholoride reduces the serum calcium concentration in inoperable parathyroid carcinoma. J Clin Endocrinol Metab 2007; 92: 3803-3808.

6 Bukowski RM, Sheeler L, Cunningham J, Esselstyn C. Successful combination chemotherapy for metastatic parathyroid cancer. Arch Intern Med 1984; 144: 399-400.

7 Bradwell AR, Harvey TC. Control of hypercalcaemia of parathyroid carcinoma by immunisation. Lancet 1999; 353: 370-373.

8 Burkiewicz JS, Scarpace SL, Bruce SP. Denosumab in osteoporosis and oncology. Ann Pharmacother 2009; 43: 1445-1455.

9 Stopeck AT, Lipton A, Body JJ, et al. Denosumab compared with zoledronic acid for the treatment of bone metastases in patients with advanced breast cancer: a randomized, double-blind study. J Clin Oncol 2010; 28: 5132-5139.

10 Henry DH, Costa L, Goldwasser F, et al. Randomized, double-blind study of denosumab versus zoledronic acid in the treatment of bone metastases in patients with advanced cancer (excluding breast and prostate cancer) or multiple myeloma. J Clin Oncol 2011; 29: 1125-1132.

11 Falchetti A, Franchi A, Bordi C, et al. Azidothymidine induces apoptosis and inhibits cell growth and telomerase activity of human parathyroid cancer cells in culture. J Bone Miner Res 2005; 20: 410-418. 